\title{
Nanotechnology in Neurology-Current Status and Future Possibilities
}

\author{
James M Provenzale, MD¹ and Aaron M Mohs, $\mathrm{PhD}^{2}$ \\ 1. Professor of Radiology, Department of Radiology, Duke University Medical Center, and Professor of Radiology, Oncology, and Biomedical Engineering, \\ Departments of Radiology, Oncology, and Biomedical Engineering, Emory University School of Medicine; 2. Distinguished Fellow, Department of Biomedical \\ Engineering, Emory-Georgia Tech Center for Cancer Nanotechnology Excellence
}

\begin{abstract}
The field of nanomedicine is rapidly emerging and will provide many novel methods for diagnosis and treatment. In this article the applications of nanotechnology to the central nervous system (CNS) will be described. Nanotechnology provides many potential solutions to various problems encountered in CNS diseases. Specifically, nanomedicine offers the possibility of new methods of drug delivery, more sensitive and specific means for diagnosis of disease at earlier stages and assessment of treatment response, and also potential techniques for neuro-protection and neuro-engineering. In this article, information is provided on the various types of nanoparticles involved in medical applications, the principles of nanoparticle delivery and targeting, and both in vivo and ex vivo uses of nanoscale materials.
\end{abstract}

\section{Keywords}

Nanoparticle, iron oxide, quantum dot, nanomedicine, drug, delivery, neuroprotection

Disclosure: The authors have no conflicts of interest to declare.

Received: May 5, 2010 Accepted: June 28, 2010 Citation: US Neurology, 2010;6(1):12-7 DOI: 10.17925/USN.2010.06.01.12

Correspondence: James M Provenzale, MD, Department of Radiology, Emory University School of Medicine, 1364 Clifton Road, Atlanta, GA 30322. E: prove001@mc.duke.edu

The term nanomedicine refers to a field in which the advances of nanotechnology are applied to health and medical diseases. This rapidly growing field is based on the remarkable advances being made in nanotechnology, a field in which materials (e.g. nanoparticles) and devices that have a size on the nanometer scale are developed. ${ }^{1}$ The relative scale of nanometer-size materials is important to understand when discussing nanomedicine. As a useful example, the diameter of a typical nanoparticle in medical use is in the order of 10-100 nanometers $(\mathrm{nm})$, which is very similar to the size of most viruses and much smaller than a cell. As will be clear from the following discussion, the extremely small size of nanoparticles and other materials on the nano-scale allows for types of interactions with living tissue that are impossible with larger materials.

Nanotechnology has a number of applications to the central nervous system (CNS), including new possibilities for the diagnosis of CNS diseases, novel methods of drug delivery, and, perhaps most intriguingly, innovative methods of regenerating CNS tissues. This article will introduce the reader to the various types of nanoparticles, the principles of nanoparticle delivery and targeting, and in vivo and ex vivo uses of nanoscale materials.

\section{Introduction to Types of Nanoscale Devices}

An explanation of the types of nanoscale devices is important in order to understand their unique properties, benefits, and limitations. As a result of space limitations, this article will focus solely on nanoparticles rather than many other types of nanomaterials that are described in more detail elsewhere. ${ }^{2}$ Throughout this discussion, the reader should be aware that the effects of nanomaterials on living human tissue is still a matter of active investigation; although many nanomaterials are believed to be safe for use in the human body, much work remains to understand the toxicology of various materials. ${ }^{3}$

\section{Liposomes and Micelles}

Liposomes are the form of nanoparticle that has found the widest use to date in actual medical applications. These particles consist of two major components: an aqueous core and a surrounding phospholipid bilayer membrane. The aqueous core provides an inner compartment in which a cargo, such as a water-soluble drug, can be carried. The phospholipid bilayer membrane provides a protective coating that insulates the contents of the inner core from degradation as well as from release of contents at unintended sites. ${ }^{4}$ As such, liposomes can be designed to provide a timed release at their intended target depending on local physiological conditions (e.g. $\mathrm{pH})^{5}$ or controlled by external stimuli (such as heat or light) applied by an operator. ${ }^{6}$

Liposomes are already in clinical use in humans. For instance, liposomes containing doxorubicin have been approved by the US Food and Drug Administration (FDA) for use in ovarian cancer and multiple myeloma. In addition, liposomal formulations of chemotherapy are under investigation for primary and secondary brain tumors. ${ }^{8}$

Micelles have some similarities to liposomes in that they also provide a protective inner environment that allows sequestration of a 
cargo that would otherwise be exposed to various physiological environments that might lead to degradation. Micelles are spherical in shape and, in addition to the hydrophobic inner core, which helps solubilize hydrophobic drugs, they have a hydrophilic shell. The hydrophilic outer component is one feature that may allow for delivery of micelles across the blood-brain barrier (BBB). ${ }^{9}$ As will be outlined below, transit across the $\mathrm{BBB}$ is one of the major obstacles for delivery of nanoparticles for CNS diseases. ${ }^{10}$

\section{Nanoshells}

One form of nanoparticles, termed nanoshells, is composed of an outer thin metal shell surrounding a spherical dielectric core. ${ }^{11}$ The relative dimensions of the core and shell can be designed in such a manner that the nanoshells can either scatter or absorb light at specific wavelengths. As such, a nanoshell of one composition can serve as an imaging agent (by virtue of light-scattering properties) ${ }^{12}$ while one with another composition can serve as a thermal ablation agent. ${ }^{11}$ This latter property is discussed later in this article in the section discussing therapy.

\section{Nanotubes}

Another important type of nanoparticle is the nanotube, which is a hollow cylindrical molecule usually made of a single element; the structures have a broad range of electrical, elastic, and thermal properties. Carbon is the element most commonly used; as a result carbon nanotubes are the form of nanotube most commonly used in medical applications. Carbon nanotubes are nanostructures composed of graphene sheets; the sheets are rolled to form cylindrical shapes. ${ }^{13}$ Carbon nanotubes can be single-walled or multiwalled and can be adapted for a variety of both in vivo and ex vivo uses. In particular, they gained attention as potential service as nanoscaffolds (described in the section below on drug delivery). The ex vivo uses of nanotubes to serve as a model of neuronal circuitry is discussed in more detail later in this article.

\section{Iron Oxide}

Iron oxide (IO) nanoparticles consist of two essential components: a metal core and surface coating. IO cores are composed of insoluble ferrites and thus require surfaces that render them water-soluble. An extensive array of molecules has been used to solubilize 10 cores. Polymeric coatings to stabilize 10 have been extensively studied, including both natural and synthetic polymers. Natural polymers, including dextran and chitosan, and synthetic polymers, especially poly(ethylene glycol), are among the most common polymers for stabilizing IO particles. ${ }^{14}$ Alternatively, inorganic materials, e.g. silica, are also used to stabilize 10 nanoparticles. ${ }^{15}$ The most significant advantage of these coatings is that they minimize macrophage uptake and thus increase blood circulation times. Each of these surface-coating methodologies provides a means to covalently attach surface ligands for targeting and therapy. ${ }^{15}$

Due to their nano-scale ferromagnetic core size, IO nanoparticles do not have permanent magnetism and are thus affected by an external magnetic field that renders them suitable as a magnetic resonance (MR) contrast agents. IO nanoparticles that are very small, termed ultrasmall superparamagnetic IO (USPIO) nanoparticles, have a core size of $<10 \mathrm{~nm}$

\section{Figure 1: Use of Nanoscaffolds for a Central Nervous System Drug in an Attempt to Avoid Systemic Side Effects of Drugs}
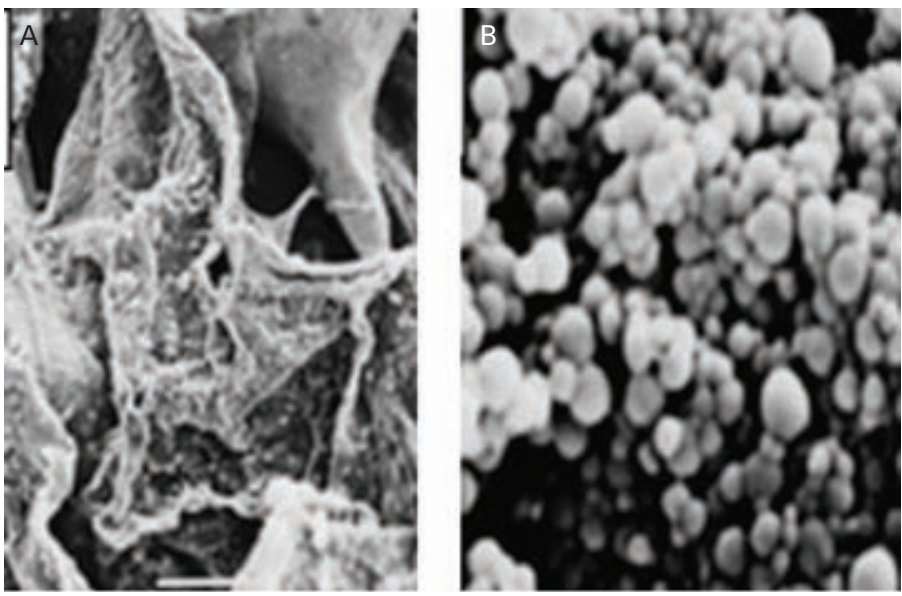

A: Scanning electron microscope image of nanoscaffold surface onto which dopamine-containing nanoparticles can be loaded. $(1 \mathrm{~mm}=0.5 \mu \mathrm{m}) ; \mathrm{B}$ : Scanning electron microscope image of nanoparticles loaded with dopamine. Reproduced from Pillay et al., $2009^{49}$ with permission.

and are $<50 \mathrm{~nm}$ after surface coating. ${ }^{16}$ USPIO nanoparticles have high longitudinal and transverse relaxivity producing an increase in signal intensity on T1-weighted images similar to the positive enhancement from gadolinium (III) complexes, and decrease MR signal on images from T2*-weighted pulse sequences. ${ }^{17,18}$

\section{Gold Nanoparticles}

Gold nanoparticles are composed of nanoscale clusters of gold atoms prepared from the reduction of gold salts. ${ }^{19}$ Bare gold nanoparticles, i.e. those with hydrophobic or low-molecular-weight stabilizing agents (e.g. citric acid) on the surface, are not stable in aqueous buffers. Stabilizing ligands, often containing a thiol group (a sulfur-hydrogen bond), are used to improve the aqueous colloidal stability of gold nanoparticles. The ease and versatility of this chemistry allows them to be coated with a variety of surface ligands and biological targeting moieties. ${ }^{20,21}$

Owing to shifting of their surface plasmon resonance (SPR) band, gold nanoparticles can be used for colorimetric assays. ${ }^{22}$ Gold nanoparticles are red in solution due to the plasmon band. However, when gold nanoparticles are aggregated the solution turns blue due to interactions of the surface plasmons and aggregate scattering. ${ }^{23}$ By controlling the aggregation of gold nanoparticles, researchers have been able to develop highly sensitive assays for detection of an array of biomolecules. ${ }^{24-26}$

Surface-enhanced Raman spectroscopy (SERS) is a second attribute of gold nanoparticles that has been exploited for sensitive biological sensing. By adsorbing 'reporter' molecules onto a metal surface, the reporter signal can be enhanced by a factor as large as 1014-1015, which allows for single-molecule detection. ${ }^{27,28}$ SERS nanoparticles have been used for the in vitro detection of proteins, viruses, and microorganisms. ${ }^{29}$ SERS nanoparticles also have a large potential for in vivo applications since there is low background interference. ${ }^{30}$ 
Figure 2: Illustration of Convection-enhanced Delivery of Liposomes for Potential Therapeutic Applications for Central Nervous System Diseases

A

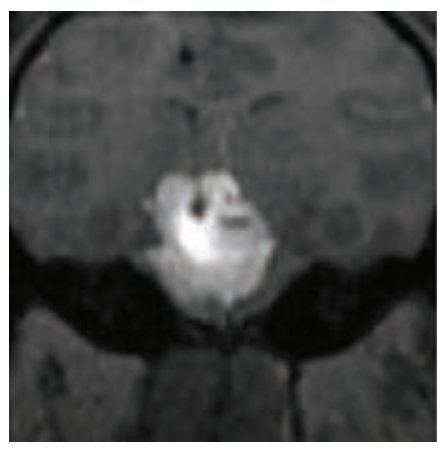

B

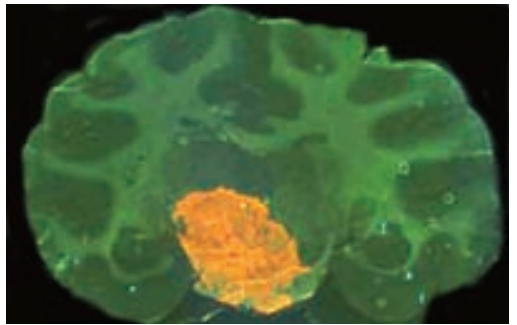

In this example, liposomes have been loaded with gadolinium-based magnetic resonance (MR) contrast material to allow distribution volume to be assessed using MR imaging. Other liposomes have been loaded with rhodamine to allow fluorescence imaging of necropsy specimens. A: Coronal T1-weighted MR image shows bright signal consistent with liposome distribution within the brainstem and adjacent regions; B: Fluorescence image obtained in coronal brain section after necropsy shows distribution of rhodamine-laden liposomes (in red) which were injected in concert with the gadolinium-based MR contrast agent liposomes. Reproduced from Krauze et al., $2008^{52}$ with permission.

\section{Quantum Dots}

Quantum dots (QDS) are semi-conductor nanocrystals composed of a metallic core and an outer shell that have size and compositiondependent fluorescent emission. ${ }^{31,32}$ QD cores and shells are commonly composed of cadmium, zinc, sulfide, selenide, and telluride. ${ }^{33}$ The shell layer (e.g. zinc sulfide) is critical to QDs used for biomedical applications because it reduces oxidation of the quantum dot, thus preserving strong fluorescence emission and minimizing the release of potentially toxic elements. ${ }^{34}$ The fluorescence emission of QDS can be finely tuned by chemical methods and physical filtering. Furthermore, computational methods allow different emission wavelengths of QDS to be easily separated. Thus, QDs are an ideal candidate for multiplexed imaging. Multiplexed imaging refers to the ability to image several biomarkers simultaneously. In this case, a different QD is targeted against each biomarker, via various conjugation techniques, to yield important quantitative data on the presence of each biomarker. ${ }^{35,36}$

QDs are often composed of heavy metals, which has elicited concern over their biocompatibility, especially for eventual in vivo applications and the environmental risks posed by QD synthesis and disposal. ${ }^{37-39}$ Two primary approaches have been undertaken to minimize the risk posed by QDs. It has been hypothesized that if QDs are kept smaller than the renal threshold, QDS can rapidly clear from the body via urinary excretion and will pose little toxicity risk. Investigations on have estimated that a size of $<5 \mathrm{~nm}$ would facilitate rapid clearance..$^{40}$ Alternatively removing or minimising the amount of heavy metals contained in QDs reduces the chances of potential toxic exposure. Significant effort has been placed in developing sizeminimized or cadmium-reduced QDs while retaining their favorable optical properties. ${ }^{41,42}$

\section{Principles of Nanoparticle Delivery and Targeting Biodistribution}

A major issue of uses of nanoparticles is delivery to sites of interest and avoidance of unintended targets (so-called non-target delivery), which are problems shared by approved contrast agents and drugs in daily use. However, when administered via intravenous injection, nanoparticles also face a major impediment that is now encountered with approved drugs and contrast agents, i.e. the high likelihood of sequestration by the reticuloendothelial system. As a means of avoiding this consequence, polyethylene glycol molecules are commonly attached to the surface of nanoparticles, which allows them to transit through the liver and spleen without being sequestered.

Another issue faced during intravenous infusion of nanoparticles intended for delivery to the brain is inability to cross an intact BBB. This barrier serves to exclude lipophilic molecules and polar or ionized water-soluble molecules. ${ }^{43}$ As a result, in many instances, nanoparticle delivery may be limited to brain regions in which the BBB is interrupted. However, in some cases, nanoparticles have been shown to cross not only into portions of the brain in which the BBB was altered but also in regions in which this barrier was intact $B B B .^{44}$

A number of methods have been developed to attempt to promote nanoparticle transport across an intact BBB. One example is the poly(butylcyanoacrylate) nanoparticle; this nanoparticle is coated with polysorbate 80 and on that basis adsorbs apolipoproteins $B$ and $E .{ }^{45} A s$ a result, transport via receptor-mediated endocytosis by brain capillary endothelial cells is promoted..$^{46,47}$

\section{Targeting of Nanoparticles}

One major advantage of nanoparticles is the fact that they can be directed against specific molecular targets; thus, they offer the promise of more sensitive and specific diagnosis of abnormal tissue. In general, this property is enabled by attaching a molecule (e.g. one or more antibodies) to the nanoparticle surface that has an affinity for some feature (e.g. a surface receptor) on the entity that is intended to be studied or treated. This feature can allow nanoparticles to be deposited in specific tissues (e.g. within a tumor). In the case of imaging agents, higher specificity and a higher signal-to background ratio is attained. In the case of therapy-delivery vehicles, non-target delivery (with the attendant drawback of systemic side effects) is minimized.

\section{In Vivo Uses of Nanoscale Materials Diagnosis \\ In Vivo Imaging}

The targeting ability of nanoparticles provides them with a number of advantages compared with conventional contrast agents and may render them able to more sensitively detect a number of disease entities. Examples include earlier detection of tumor, demyelinating conditions, and degenerative diseases. For instance, iron oxide 
nanoparticles targeted against glioma cells have been developed that employ the surface-bound peptide chlorotoxin, thereby becoming able to detect any tumor expressing membrane-bound metalloproteinase-2. ${ }^{17}$ The superparamagnetic properties of the iron oxide nanoparticles allow them to be detected with a high degree of sensitivity on MR imaging. As another example, nanoparticles that can serve as either optical imaging agents or MR imaging agents have been targeted against polysialic acid, a carbohydrate that is important in a variety of neural pathways but is also a marker of a number of tumors, such as neuroblastoma and lung carcinoma. ${ }^{48}$ The optical properties of these particles are provided by rhodamine dye-dopa silica and the MR-imaging properties are provided by iron oxide nanoparticles, to which the silica is conjugated.

\section{Therapy}

\section{Drug Delivery Vehicles}

One proposed solution for bypassing the BBB in order to provide drug therapy is implantation of a site-specific intracranial device that could slowly release drugs. As an example, one such proposed delivery vehicle is designed for treatment of Parkinson's disease, i.e. a nanoscaffold onto which are imbedded nanoparticles containing dopamine for therapy of Parkinson's disease (see Figure 1). ${ }^{49}$ By implanting the nanoscaffolds within brain tissue, the systemic effects of dopamine are avoided. The scaffold is composed of cross-linked alginate into which dopamine-loaded nanoparticles composed of cellulose acetate phthalate are embedded. The nanoparticles are approximately $200 \mathrm{~nm}$ in size. The investigators have studied the use of the nanoscaffolds device in vivo in rats. After implantation of the device in the frontal lobe, dopamine levels in the cerebrospinal fluid (CSF) and plasma were measured serially over the period of many days. Peak dopamine levels in the CSF and plasma both peaked at three days. The peak CSF concentration represented $28 \%$ of the total dopamine available from the nanoscaffolds; thereafter, CSF dopamine levels remained at a plateau. However, the peak plasma concentration was only approximately $1 \%$ of the available dopamine. Low plasma levels were seen over the 30-day observational period, which is considered beneficial from the standpoint of minimizing drug side effects.

As the BBB presents a formidable impediment to delivery of various therapeutic substances to the CNS, various attempts have been made to bypass the BBB by direct injection of delivery vehicles containing chemotherapy or other agents. As an example, investigators have focused on convection-enhanced delivery (CED) for introduction of materials into the brain. CED is a relatively new technique in which therapeutic substances are infused through an indwelling catheter directly into the interstitial spaces of brain parenchyma via a fluid pressure gradient. Using this technique, therapeutic molecules or carriers for such molecules can be directly instilled into the brain. Examples of such molecules include chemotherapeutic agents and factors intended to reverse effects of neurodegenerative diseases. . $^{50,51}$

Investigators have examined CED as a route for the administration of nanoparticles carrying various types of therapeutic drugs. In one example, researchers studied CED administration of liposomes
Figure 3: Illustration of the Use of Nanofactories for Delivery of Therapeutic Agents

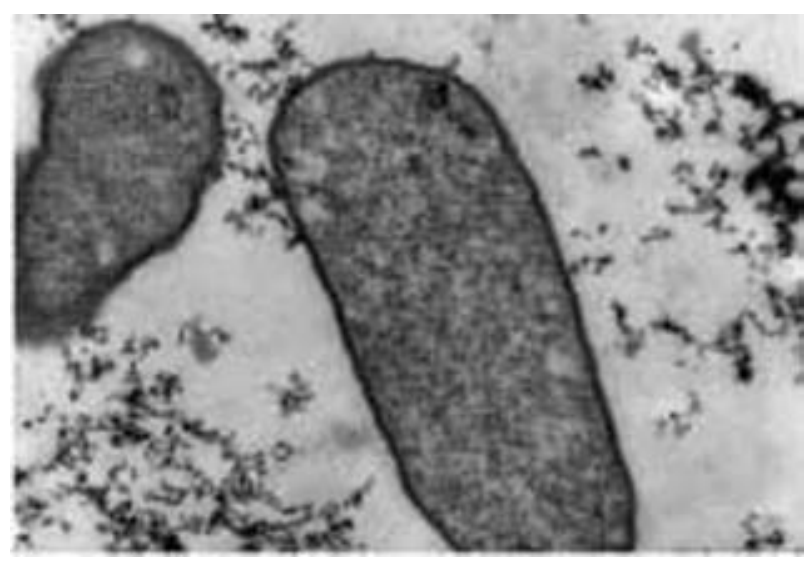

In this example, investigators have used nanofactories to synthesize and deliver the signal molecule autoinducer Al-2, to the surface of Escherichia coli cells. Transmission electron microscope image shows nanofactories (seen as small black structures) attaching to walls of E. coli target cells.

Reproduced from Fernandes et al., 2007 ${ }^{53}$ with permission.

Figure 4: Illustration of Use of Neural Conduits for Repair of Peripheral Nerve Injury

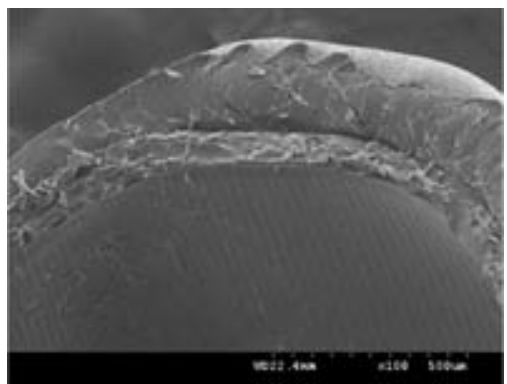

Scanning electron micrograph shows neural conduit composed of chitosan-gold nanocomposites material. The inner surface of the conduit has a micro pattern intended to direct cell regrowth. The conduit can be seeded with biologically active materials, e.g. neuronal stem cells, to promote repair by such means as axonal regeneration. Reproduced from Lin et al., $2008^{54}$ with permission.

containing a gadolinium-based MR contrast agent into the primate brain so that intracerebral distribution of the nanoparticles could be assessed using MR imaging (see Figure 2). ${ }^{52}$ For this study, liposomes containing three doses of MR contrast material were infused, with each primate receiving one of the three doses. Each animal underwent up to three infusions into the left putamen, each separated by four weeks. A variety of infusion volumes was used. Along with the gadolinium-laden nanoparticles, the investigators infused liposomes containing rhodamine that would allow fluorescence imaging of necropsy specimens. The goal was to determine whether distribution of the liposomes within various structures could be predicted, whether repeated procedures produce the same results, and whether any histological damage resulted. In this study, the liposomes did not contain a therapeutic drug; instead, the study aimed solely to determine the distribution patterns of the liposomes to prepare for subsequent trials using liposomes containing drugs. The study showed that MR signal after infusion was proportional to concentration of gadolinium within the liposomes, with higher signal being seen in animals treated 


\section{Figure 5: Depiction of the Use of Carbon Nanotubes as a Means to Better Understand the Complexities of Neuronal Circuits}

A

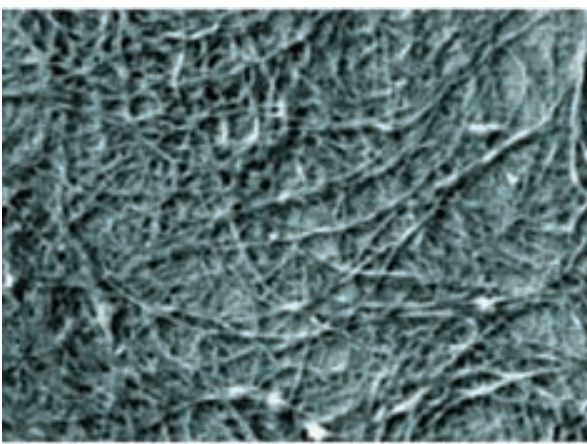

B

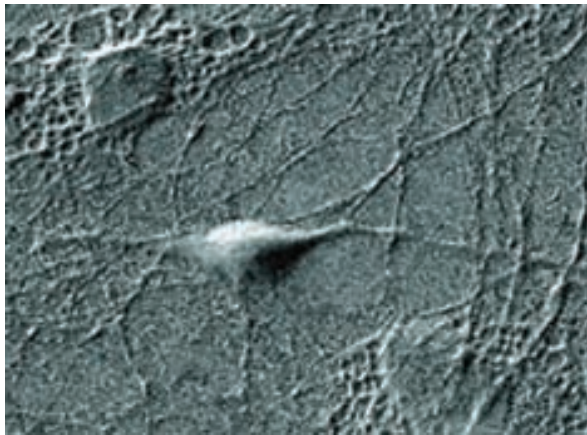

In this example, culture neurons are grown on carbon nanotubes for many weeks and organize into functional networks. A: Scanning electron micrograph image shows a network of carbon nanotubes onto which cell culture will be grown; B: Scanning electron micrograph shows neurons growing in close proximity to carbon nanotubes. Neuronal-carbon nanotube electrical circuits develop which can then serve as a model for understanding the physiologic and chemical structure of the neuronal circuit.

Reproduced from Giugliano et al., $2008^{13}$ with permission.

with liposomes having the higher concentration of MR contrast material. The distribution of MR signal extended beyond the putamen to include the adjacent brainstem and corona radiata, which was confirmed on fluorescence imaging (see Figure 2). The distribution of liposomes was found to be highly reproducible. Histologic examination showed mild myelin pallor on the infused side of the brain and no evidence of immunoreactivity beyond that expected for age.

\section{Nanofactories}

One innovative method for providing important substances to cells is to manufacture those substances (from precursor molecules) at the cell surface by means of a nanofactory. Such a process would allow prescribed amounts of molecules to be synthesized at a pre-established time and avoid many of the issues involved in delivery by producing them at the site of the intended target.

As an example, researchers have synthesized and delivered the signal molecule autoinducer Al-2, to the surface of Escherichia coli cells using such a nanofactory (see Figure 3). ${ }^{53}$ The first step in the assembly of the nanofactory is co-precipitation of nanoparticles of iron salts and the biopolymer chitosan, which serves as a molecular scaffold. The chitosan biopolymer has two roles: to provide the nanofactory with the means to bind to the cell surface and to provide a means of attachment of important enzymes to the nanofactory. The next step is covalent attachment of the enzymes (in this case, two E. coli Al-2 synthases) onto the chitosan scaffold. These enzymes have the ability to manufacture the Al-2 signaling molecule. In this manner, the nanofactory is able to manufacture the needed molecule directly at the cell surface.

\section{Neuro-engineering}

One of the more intriguing possibilities offered by nanotechnology as applied to the CNS is that of re-engineering brain and spine tissue. Nanoparticles offer the capability, at least in theory, of delivering materials needed for regeneration of diseased or injured tissue and directing subsequent repair (i.e. so-called 'neuro-engineering'). An example of such a process is described below.

\section{Nerve Regrowth via Conduits}

Traumatic peripheral nerve injuries are commonly treated in neurosurgical practice. The rate at which peripheral nerves spontaneously repair is very slow and can be accompanied by a number of poor results such as impaired nerve conduction and formation of aberrant conduction pathways. Surgical repair is also often difficult; in cases of severe nerve transection, the two nerve stumps may be widely separated and in those instances a nerve graft (usually using autologous nerve) is needed. Conduits composed of nanomaterials offer a potential alternative means for nerve repair. One such conduit is composed of chitosan, a polysaccharide similar in nature to glycosaminoglycans, which renders the graft biodegradable (see Figure 4). ${ }^{54}$ The relatively good mechanical strength of the chitosan is buttressed by the addition of gold nanoparticles. To enhance the reparative process, the conduits are seeded with neuronal stem cells; furthermore, the inner surface of the conduit is grooved to promote alignment of growth of the stem cells along the Iong axis of the graft. Compared with conduits that are not prepared with neuronal stem cells, those seeded with neuronal stem cells were shown to produce a greater number of regenerated axons and a greater area of regeneration.

\section{Ex Vivo Uses of Nanoscale Materials}

\section{Imaging of Biomarkers in Pathology Specimens}

One use of nanoparticles that shows much promise is more accurate diagnosis of tumor tissue in pathology specimens. For instance, the use of QDS for multiplex imaging of various forms of cancer in histological samples is an area of active investigation. In particular, the multiplexing capability, i.e. the ability to depict the presence of multiple biomarkers (e.g. multiple types of tumor receptors in a specimen), offered by QDS is considered a potentially important advance over standard Immunohistochemical techniques because it may better depict the heterogeneous nature of a tumor specimen as reflected in the complex genetic, molecular, and structural changes that are present. ${ }^{36}$

\section{Uses of Nanoparticles in Cell Culture and Model Neuronal Circuits}

One of the more interesting features of carbon nanotubes is that they can detect neuronal activity and also emit electrical stimulation. Hence, they have the possibility of serving as interfaces with neurons. As one example of their use, researchers have used such nanoparticles as a 
means to enhance and direct the growth and morphology of neurons after CNS injury. ${ }^{13}$ By chemically modifying the surface of the nanotubes, the nanotubes can serve to promote neuronal regrowth. Furthermore, such nanotubes can be incorporated into neuronal cell cultures; after establishment of neuron-carbon nanotube electrical coupling, and through chemical or pharmacologic modulation, these nanoparticles may provide a means to better understand the complexities of neuronal circuits (see Figure 5).

\section{Summary}

As this article has indicated, many applications of nanomaterials for use in the CNS have already been developed in cell culture and small animals systems. The spectrum of possible applications for use in the CNS is wide and includes new forms of diagnosis and therapy, potential uses for tissue regeneration, and novel methods for understanding the behavior of cells and neuronal circuits ex vivo.

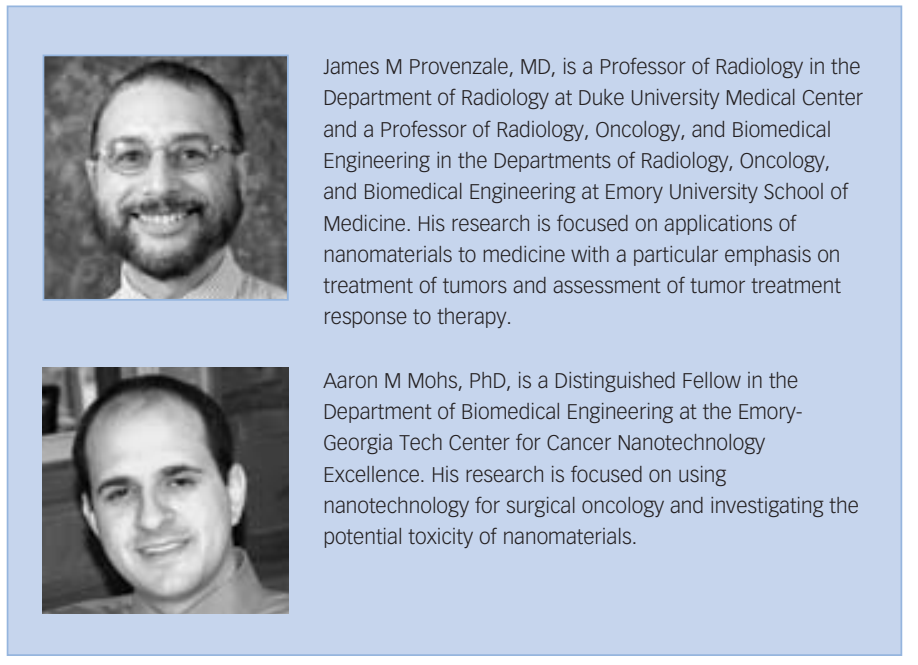

Neurobiol, 2004:30:456-71.

1. Leary SP, Liu CY, Apuzzo ML, Neurosurgery, 2006;58: 1009-26.

2. Ferrari M, Nat Rev Cancer, 2005;5:161-71.

3. Wiesner MR, Lowry GV, Alvarez P, et al., Environ Sci Technol, 2006;40:4336-45

4. Ruiz MA, Clares B, Morales ME, et al., Drug Dev Ind Pharm, 2008;34:1269-76

5. Gu J, Cheng WP, Liu J, et al., Biomacromolecules, 2008:9:255-62.

6. VanderMeulen DL, Misra P, Michael J, et al., Photochem Photobiol, 1992;56:325-32.

7. Rose PG, Oncologist, 2005:10:205-14.

8. Caraglia M, Addeo R, Costanzo R, et al., Cancer Chemother Pharmacol, 2006:57:34-9.

9. Liu L, Venkatraman SS, Yang YY, et al., Biopolymers, 2008;90:617-23.

10. Provenzale JM, Mukundan S, Dewhirst M, AIR Am J Roentgenol, 2005;185:763-7.

11. Bernardi RJ, Lowery AR, Thompson PA, et al., I Neurooncol, 2008;86:165-72.

12. Loo C, Lin A, Hirsch L, et al., Technol Cancer Res Treat, 2004;3:33-40

13. Giugliano M, Prato M, Ballerini L, Drug Discov Today Dis Models, 2008;5:37-43

14. Laurent S, Forge D, Port M, et al., Chem Rev, 2008:108:2064-2110

15. Liu Q, Xu Z, Finch JA, et al., Chemistry of Materials, 1998:10:3936-40

16. Corot C, Robert $P$, Idee JM, et al., Adv Drug Deliv Rev, 2006:58:1471-1504.

17. Sun C, Veiseh O, Gunn J, et al., Small, 2008;4:372-9.

18. Neuwelt EA, Varallyay $P$, Bago AG, et al., Neuropathol App
19. Ghosh P, Han G, De M, et al., Adv Drug Deliv Rev, 2008:60:1307-15.

20. Dixit $\mathrm{V}$, Van den Bossche J, Sherman DM, et al., Bioconjug Chem, 2006:17:603-9.

21. Paciotti GF, Myer L, Weinreich D, et al., Drug Deliv, 2004;11:169-83

22. Daniel MC, Astruc D, Chem Rev, 2004;104:293-346.

23. Rosi NL, Mirkin CA, Chem Rev, 2005;105:1547-62.

24. He L, Musick MD, Nicewarner SR, et al., J Am Chem Soc, 2000;122:9071-7.

25. Pavlov V, Xiao Y, Shlyahovsky B, et al., J Am Chem Soc, 2004;126:11768-9.

26. Elghanian R, Storhoff JJ, Mucic RC, et al., Science, 1997;277:1078-81.

27. Nie S, Emory SR, Science, 1997;275:1102-6

28. Cao YC, Jin R, Mirkin CA, Science, 2002;297:1536-40

29. Porter MD, Lipert RJ, Siperko LM, et al., Chem Soc Rev, 2008;37:1001-11.

30. Qian X, Peng X-H, Ansari DO, et al., Nat Biotech, 2008:26:83-90.

31. Bruchez M Jr, Moronne M, Gin P, et al., science, 1998;281:2013-16.

32. Smith AM, Duan $\mathrm{H}$, Mohs AM, et al., Adv Drug Deliv Rev, 2008;60:1226-40.

33. Murray CB, Norris DJ, Bawendi MG, J Am Chem SOC, 1993;115:8706-15

34. Dabbousi BO, Rodriguez-Viejo J, Mikulec FV, et al., J Phys Chem, 1997;101:9463-75.

35. Xing Y, Chaudry Q, Shen C, et al., Nat Protocols, 2007:2:1152-65

36. Liu J, Lau SK, Varma VA, et al., ACS Nano, 2010;4:2755-65.
37. Nel A, Xia T, Madler L et al, science, 2006:311:62-7.

38. Yang RS, Chang LW, Wu JP, et al., Environ Health Perspect, 2007:115:1339-43.

39. Hardman R, Environ Health Perspect, 2006;114:165-72.

40. Choi HS, Liu W, Misra P, et al., Nat Biotechnol, 2007;25:1165-70

41. Pradhan N, Battaglia DM, Liu Y, et al., Nano Lett, 2007;7:312-17.

42. Smith AM, Mohs AM, Nie S, Nat Nanotechnol, 2009;4:56-63.

43. Bart J, Groen $\mathrm{HJ}$, Hendrikse $\mathrm{NH}$, et al., Cancer Treat Rev, 2000;26:449-62.

44. Calvo P, Gouritin B, Villarroya $\mathrm{H}$, et al., Eur J Neurosci, 2002;15:1317-26

45. Gulyaev AE, Gelperina SE, Skidan IN, et al., Pharm Res, 1999;16:1564-9.

46. Olbrich C, Gessner A, Kayser O, et al., J Drug Target, 2002;10:387-96

47. Alyaudtin RN, Reichel A, Lobenberg R, et al., J Drug Target, 2001;9:209-21

48. Lee JH, Jun YW, Yeon SI, et al., Angew Chem Int Ed Engl 2006;45:8160-62

49. Pillay S, Pillay V, Choonara YE, et al., Int J Pharm, 2009;382:277-90

50. Gill SS, Patel NK, Hotton GR, et al., Nat Med, 2003;9:589-95

51. Sampson JH, Raghavan R, Provenzale JM, et al., AJR Am J Roentgenol, 2007;188:703-9.

52. Krauze MT, Vandenberg SR, Yamashita Y, et al., Exp Neurol, 2008:210:638-44.

53. Fernandes R, Tsao CY, Hashimoto Y, et al., Metab Eng, 2007:9:228-39.

54. Lin $\mathrm{YL}$, Jen JC, Hsu SH, et al., Surg Neurol, 2008;70 (Suppl. 1):S1:9-18. 